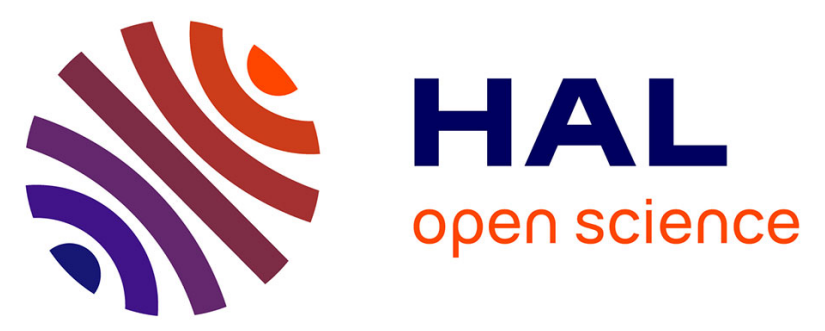

\title{
Dihydroxylation of four- and five-ring aromatic hydrocarbons by the naphthalene dioxygenase from Sphingomonas CHY-1
}

\author{
Yves Jouanneau, Christine Meyer, Nicolas Duraffourg
}

\section{To cite this version:}

Yves Jouanneau, Christine Meyer, Nicolas Duraffourg. Dihydroxylation of four- and five-ring aromatic hydrocarbons by the naphthalene dioxygenase from Sphingomonas CHY-1. Applied Microbiology and Biotechnology, 2016, 100 (3), pp.1253-1263. 10.1007/s00253-015-7050-y . hal-01260091

\section{HAL Id: hal-01260091 \\ https://hal.science/hal-01260091}

Submitted on 21 Jan 2016

HAL is a multi-disciplinary open access archive for the deposit and dissemination of scientific research documents, whether they are published or not. The documents may come from teaching and research institutions in France or abroad, or from public or private research centers.
L'archive ouverte pluridisciplinaire $\mathbf{H A L}$, est destinée au dépôt et à la diffusion de documents scientifiques de niveau recherche, publiés ou non, émanant des établissements d'enseignement et de recherche français ou étrangers, des laboratoires publics ou privés. 
1 Dihydroxylation of four- and five-ring aromatic hydrocarbons by the naphthalene

2 dioxygenase from Sphingomonas CHY-1

3

4

5

6 Yves Jouanneau ${ }^{1,2,3}$, Christine Meyer ${ }^{1,2,3}$, and Nicolas Duraffourg ${ }^{1,2,3}$

7

$8{ }^{1}$ CEA, DSV, iRTSV, Laboratoire de Chimie et Biologie des Métaux, F-38054 Grenoble, 9 France.

$10 \quad{ }^{2}$ CNRS, UMR 5249, Grenoble, France.

$11{ }^{3}$ Univ. Grenoble Alpes, F-38000 Grenoble, France

12

13

14 Running title : Dioxygenation of 4- and 5-ring PAHs

15

16

17 Corresponding author:

$18 \quad$ Yves Jouanneau

19

LCBM/iRTSV, CEA-Grenoble

F-38054 Grenoble Cedex 9, France.

21

Tel. : 33 (0)4 387843 10; Fax : 33 (0)4 38785487

22

Email : yves.jouanneau@cea.fr

23 


\section{ABSTRACT}

2 The naphthalene dioxygenase from Sphingomonas CHY-1 exhibits extremely broad substrate 3 specificity toward polycyclic aromatic hydrocarbons (PAHs). In a previous study, the

4 catalytic rates of oxidation of 9 PAHs were determined using the purified dioxygenase, but 5 the oxidation products formed from 4- to 5-ring hydrocarbons were incompletely 6 characterized. Here, we reexamined PAH oxygenation reactions using Escherichia coli 7 recombinant cells overproducing strain CHY-1 dioxygenase. Hydroxylated products 8 generated by the dioxygenase were purified, and characterized by means of GC-MS, UV 9 absorbance as well as ${ }^{1} \mathrm{H}$ and ${ }^{13} \mathrm{C}$-NMR spectroscopy. Fluoranthene was converted to 3 10 dihydrodiols, the most abundant of which was identified as cis-7,8-dihydroxy-7,811 dihydrofluoranthene. This diol turned out to be highly unstable, converting to 812 hydroxyfluoranthene by spontaneous dehydration. The dioxygenase also catalyzed dihydroxylations on the $\mathrm{C} 2-\mathrm{C} 3$, and presumably the $\mathrm{C} 1-\mathrm{C} 2$ positions, although at much lower rates. Benz[a]anthracene was converted into three dihydrodiols, hydroxylated in positions C1-C2, C8-C9 and C10-C11, and one bis-cis-dihydrodiol. The latter compound was identified as cis,cis-1,2,10,11-tetrahydroxy-1,2,10,11-tetrahydrobenz[a]anthracene, which resulted from the subsequent dioxygenation of the 1,2- or 10,11-dihydrodiols. Chrysene dioxygenation yielded a single diol identified as cis-3,4-dihydroxy-3,4-dihydrochrysene, which underwent further oxidation to give cis, cis-3,4,9,10 chrysene tetraol. Pyrene was a poor substrate for the CHY-1 dioxygenase and gave a single dihydrodiol hydroxylated on C4 and C5, whereas

21 benzo[a\} pyrene was converted to two dihydrodiols, one of which was identified as cis-9,1022 dihydrodiol. The selectivity of the dioxygenase is discussed in the light of the known 3D structure of its catalytic component, and compared to that of the few enzymes able to attack 424 and 5-ring PAHs.

25 Key words : Ring hydroxylation ; dioxygenase ; cis-dihydrodiols ; PAH ; regioselectivity 


\section{INTRODUCTION}

2 Polycyclic aromatic hydrocarbons (PAHs) are a class of hazardous hydrophobic compounds

3 that are widespread in the environment. Due to their high chemical stability and low aqueous

4 solubility, they persist for long times on contaminated sites, especially high molecular weight

5 (HMW) PAHs that are composed of 4 to 6 rings. Microbial biodegradation is mainly

6 responsible for natural attenuation and is exploited in several strategies of bioremediation

7 (Bamforth and Singleton 2005; Lu et al. 2011). PAH biodegradation pathways have been extensively studied with specialized microorganisms, most often isolated from contaminated sites. In bacteria, the catabolic pathways leading to the complete mineralization of 2- and 3ring PAHs has been described in detail (Doyle et al. 2008; Peng et al. 2008). In contrast, the degradation of HMW-PAHs is much less understood, except in a Mycobacterium strain where the metabolism of pyrene and fluoranthene has been thoroughly investigated (Kim et al. 2007; Kweon et al. 2007). The lower level of understanding of HMW-PAHs degradation, might be due to two main reasons: (i) only a few bacterial isolates were found capable of degrading such compounds, including Sphingomonads (Kunihiro et al. 2013; Lyu et al. 2014; Maeda et al. 2014; Story et al. 2001; Willison 2004) and Mycobacteria (Heitkamp et al. 1988; Krivobok et al. 2003; Schneider et al. 1996; Vila et al. 2001), and (ii) the degradation pathways are complex and often involve multiple routes (Kweon et al. 2007; Rehmann et al. 2001). In addition, some PAHs like benzo[a]pyrene are thought to be degraded through co-metabolism (Juhasz and Naidu 2000).

21 The initial step in the oxidative degradation of PAHs by bacteria is catalyzed by specific metalloenzymes called ring-hydroxylating dioxygenases (RHDs). They are multi-component enzymes, most often comprising three elements, a NAD(P)H-oxidoreductase, a ferredoxin and an oxygenase that carries the active site (Jouanneau et al. 2011; Parales and Resnick 2006). The oxygenase component is generally an hexamer $\alpha_{3} \beta_{3}$, where each alpha subunit 
1 binds a Rieske-type [2Fe-2S] cluster and a mononuclear ferrous iron center, which is the

2 catalytic site of the enzyme. The RHD enzymatic reaction involves several steps, including

3 binding of the substrate, electron transfer from $\mathrm{NAD}(\mathrm{P}) \mathrm{H}$ to the active site, dioxygen

4 activation, and hydroxylation of two adjacent carbon atoms of the substrate to give a cis-

5 dihydrodiol (Karlsson et al. 2003).

6 RHDs form a large enzyme family that has been divided into four or five subgroups, 7 essentially based on comparisons of their alpha subunit sequences (Kweon et al. 2008; Nam et 8 al. 2001). Two subgroups appeared to be composed of PAH-specific RHDs, which differ 9 depending on whether they belonged to Gram-positive or Gram-negative bacteria (Jouanneau et al. 2011; Kweon et al. 2008). Among the few RHDs able to attack HMW-PAHs, the 11 naphthalene dioxygenase from Sphingomonas CHY-1 is one of the best-characterized enzyme.

12 Its structural genes have been cloned and overexpressed in Escherichia coli, thus producing a 13 functional enzyme able to oxidize 2- to 4-ring PAHs (Demaneche et al. 2004). The RHD was 14 later purified and its catalytic activity towards 9 PAHs ranging from 2 to 5 rings was characterized with respect to reaction rates and the nature of the dihydrodiols formed 16 (Jouanneau et al. 2006). These studies showed that some 3- or 4-ring substrates like fluorene 17 and benz[a]anthracene were converted to multiple hydroxylated products. It was also observed that the dioxygenase could further oxidize some dihydrodiols generated from 4-ring PAHs to yield tetraols. However, the amounts of reaction products obtained from small-scale enzymatic assays were limited, thus preventing adequate structural characterization of the 21 hydroxylated products. In the present work, we describe the production, purification and 22 structural investigations on such products, using a E. coli strain overproducing the CHY-1 dioxygenase as a biocatalyst. We identified new oxidation products generated from pyrene and fluoranthene, and further analyzed the structure of tetraols produced from chrysene and benz[a]anthracene by NMR spectroscopy. Results are discussed by referring to the known 3D 
1 structure of the Sphingomonas CHY-1 dioxygense catalytic component (Jakoncic et al. 2 2007a; Jakoncic et al. 2007b).The regioselectivity of this enzyme is also compared to that of 3 other RHDs able to attack HMW-PAHs, which have been described thus far, essentially in 4 Sphingomonads and Mycobacteria. 


\section{Incubation of PAHs with recombinant cells overproducing strain CHY-1 RHD}

4 Escherichia coli BL21(DE3) carrying plasmids pSD9 and pEB431, has been previously 5 described (Demaneche et al. 2004)-. This strain, subsequently referred to as Bphn12, 6 overproduces the three components of the ring-hydroxylating dioxygenase from Sphingomonas CHY-1. Cultures of Bphn12 (0.8 L) were grown at $37^{\circ} \mathrm{C}$ on LB medium to an optical density of 1.5 at $600 \mathrm{~nm}$, then subjected to overnight induction with $0.2 \mathrm{mM}$ IPTG at $25^{\circ} \mathrm{C}$. Cells were harvested by centrifugation, washed and resuspended in the same volume of M9 medium containing $10 \mathrm{mM}$ glucose. Each studied PAH was then separately provided as a $0.1 \mathrm{~g} / \mathrm{L}$ solution in silicone oil (Rhodorsil 47V20, Sodipro, France), added at one fifth the volume of bacterial suspension. Biphasic cultures were incubated in 2-L flasks at $25^{\circ} \mathrm{C}$ on a rotary shaker (200 rpm) for $23 \mathrm{~h}$ ( $45 \mathrm{~h}$ for pyrene).

\section{Extraction and purification of the PAH oxidation products}

Following incubation of bacteria with $\mathrm{PAH}$, the biphasic suspension was immediately decanted to remove the oil phase, then centrifuged $15 \mathrm{~min}$ at 5,000 $\mathrm{g}$. The dihydrodiols and other relevant PAH oxidation products were recovered from the aqueous supernatant by solidphase extraction on C18 Bond Elut-cartridges (1g ; Varian). The cartridge was washed with $100 \mathrm{ml}$ of water, then eluted with $5 \mathrm{ml}$ of acetonitrile. The crude extracts thus obtained were resolved by reverse-phase HPLC and thin layer chromatography (TLC). TLC was performed on 20 x $20 \mathrm{~cm}$ silica-coated glass plates (Merck) developed with chloroform/acetone 80/20 by volume unless otherwise noted. After separation, compounds of interest were visualized under UV light and recovered from the silica by elution with $0.5 \mathrm{ml}$ acetonitrile. HPLC was 
1 appropriate wavelengths. Separation was performed on a 10 by $300 \mathrm{~mm} \mu$ Bondapak C18

2 column (Millipore Waters) run in the isocratic mode at $1.5 \mathrm{ml} / \mathrm{min}$ with a water/acetonitrile

3 mixture containing either 40 or $50 \%$ organic solvent. Benz[a]anthracene dihydrodiols, which

4 co-eluted by reverse-phase HPLC, could be separated on a 4.6 by $150 \mathrm{~mm}$ Zorbax RX-SIL

5 column (Agilent Technologies), isocratically eluted at $0.9 \mathrm{ml} / \mathrm{min}$ with a solvent made of

6 hexane/dioxane/ethanol in the 93/5/2 ratio. Purified products were stored dry or in 50\%

7 acetonitrile in sealed vials at $-20^{\circ} \mathrm{C}$.

\section{UV absorbance recording and estimation of dihydrodiol concentrations}

The UV absorbance spectra of purified PAH oxidation products were recorded on appropriate

11 dilutions in pure or 50\% acetonitrile using a HP8452 diode-array spectrophotometer (Agilent technologies). The dihydrodiol concentrations were calculated using the following absorption coefficients: $\varepsilon_{278}=57,650 \mathrm{M}^{-1} \cdot \mathrm{cm}^{-1}$ for cis-3,4-dihydroxy 3,4-dihydrochrysene (Boyd et al. $1997) ; \varepsilon_{280}=66,500 \mathrm{M}^{-1} . \mathrm{cm}^{-1}$ for cis-9,10-dihydroxy-9,10-dihydrobenzo[a]pyrene (Gibson et al. 1975); $\varepsilon_{263}=31,000 \mathrm{M}^{-1} \cdot \mathrm{cm}^{-1}$ for cis-1,2-dihydroxy-1,2-dihydrobenz[a]anthracene and $\varepsilon_{275}$ $=37,000 \mathrm{M}^{-1} \cdot \mathrm{cm}^{-1}$ for cis-10,11-dihydroxy-10,11-dihydrobenz[a]anthracene (Jerina et al. 17 1984). Pyrene 4,5-dihydrodiol was estimated by using an absorption coefficient of 98,000 $\mathrm{M}^{-}$ $18{ }^{1} . \mathrm{cm}^{-1}$ at $260 \mathrm{~nm}$, and fluoranthene 2,3-dihydrodiol was quantified using $\varepsilon_{263}=40,000 \mathrm{M}^{-}$ $19{ }^{1} \cdot \mathrm{cm}^{-1}$.

For the 7,8-fluoranthene dihydrodiol, the absorbance coefficients were determined as follows:

21 Taking advantage of the fact that the short-term fluoranthene oxidation reaction catalyzed by 22 purified PhnI produced $>98 \%$ of 7,8-dihydrodiol ((Jouanneau et al. 2006) and see Results), we carried out the complete conversion of a known amount of fluoranthene $(20 \mu \mathrm{M})$ by purified PhnI within 30 min under conditions previously described (Jouanneau et al. 2006). 
1 sulfate. After solvent evaporation, the dihydrodiol was taken up in $0.5 \mathrm{ml}$ acetonitrile and its

2 absorbance spectrum was recorded. The purity of the product and absence of residual

3 fluoranthene was checked by HPLC as described previously (Jouanneau et al. 2006).

4 Assuming a $40 \mu \mathrm{M}$ concentration in the extract, the absorbance coefficient of cis-7,8-

5 dihydrodiol was calculated to be: $\varepsilon_{334}=10,100 \mathrm{M}^{-1} \mathrm{~cm}^{-1}$ at $334 \mathrm{nmn} ; \varepsilon_{236}=29,950 \mathrm{M}^{-1} \mathrm{~cm}^{-1}$

6 ar $236 \mathrm{~nm}$ and $\varepsilon_{442}=1,370 \mathrm{M}^{-1} \mathrm{~cm}^{-1}$ at $442 \mathrm{~nm}$.

8 GC/MS analyses

9 Purified PAH oxidation products were subjected to GC/MS analysis as previously described (Jouanneau et al. 2006) with minor modifications. Briefly, samples were derivatized with bis(trimethysilyl)trifluoroacetamide : trimethylchlorosilane (99:1) from Macherey-Nagel, prior to analysis using a HP6890 gas chromatograph coupled to a HP5973 mass spectrometer

13 (Agilent Technologies). Samples $(2.5 \mu \mathrm{l})$ were injected into a capillary column (30-m long, $14 \quad 0.25-\mathrm{mm}$ internal diameter, Varian VF-5ms) using the split mode (a split ratio of 20:1 was applied). Each run was carried out at an helium flow rate of $1 \mathrm{ml} / \mathrm{min}$, with a temperature gradient from 75 to $300^{\circ} \mathrm{C}$. Mass spectra were acquired in the total ion current mode. Some samples were derivatized with N-butyl boronate (Sigma-Aldrich) prior to GC/MS analysis performed as above.

20 NMR analyses of purified dihydrodiols and tetraols

$21{ }^{1} \mathrm{H},{ }^{13} \mathrm{C}$ and ${ }^{2} \mathrm{D}-\mathrm{NMR}$ spectra were recorded at $300 \mathrm{~K}$ with a Bruker DPX 300 spectrometer 22 equipped with a QNP 5-mm probehead with a z-gradient coil for 2D experiments $\left(90^{\circ}: 11.0\right.$ $23 \mu$ s at $0 \mathrm{~dB}{ }^{1} \mathrm{H} ; 5.20 \mu \mathrm{s}$ at $\left.-6 \mathrm{~dB}{ }^{13} \mathrm{C}\right)$. Samples were dissolved in methanol-d $4\left({ }^{1} \mathrm{H} /{ }^{13} \mathrm{C} 3.30 / 49\right.$ $24 \mathrm{ppm})$ for the chrysene tetraol and fluoranthene diol, or in acetone-d6 $\left({ }^{1} \mathrm{H} /{ }^{13} \mathrm{C} 2.04 / 29.8 \mathrm{ppm}\right)$ 25 for the benz[a]anthracene tetraol. Signal assignments were determined based on recordings of 
1 gradient-assisted correlated spectroscopy (COSY) and gradient-assisted nuclear Overhauser

2 spectroscopy (NOESY) with a mixing time of $800 \mathrm{~ms}$, which were referred to as cosygp and 3 noesygp, respectively, in the Bruker library. A DEPT 135 sequence was performed to

4 complete carbon assignments. To assign carbons bearing a proton, a gradient-assisted 5 heteronuclear multiple quantum correlation (GRASP-HMQC) optimized for the detection of 6 the ${ }^{1} J_{\mathrm{CH}}(145 \mathrm{~Hz})$ with ${ }^{13} \mathrm{C}-\mathrm{GARP}$ decoupling (GARP: $85 \mu \mathrm{s}$, aq: $175 \mathrm{~ms}$ ) was used.

7 Benz[a]anthracene tetraol analysis was performed by recording gradient-assisted 8 heteronuclear multiple quantum correlation (gHMQC), double quantum filtered correlated 9 spectroscopy (DQF-COSY) and gradient-assisted nuclear Overhauser spectroscopy with 10 mixing time of 150 and $300 \mathrm{~ms}$ on a Varian INOVA 600 spectrometer using a standard $5 \mathrm{~mm}$ 11 triple resonance probehead. 
RESULTS

Biotransformation of PAHs by E. coli overproducing Sphingomonas CHY-1 RHD

Strain Bphn12 is a BL21(DE3) derivative overproducing the three components of stain CHY1 RHD namely, PhnI (oxygenase), PhnA4 (reductase) and PhnA3 (ferredoxin) (Demaneche et al. 2004). It was used as a biocatalyst to oxidize fluoranthene, benz[a]anthracene (BaA), chrysene and pyrene, as representative 4-ring PAHs, as well as beno[a]pyrene (BaP). In order to generate sufficient PAH oxidation products for subsequent analysis, $0.96 \mathrm{~L}$ biphasic cultures were separately incubated with each PAH for $23 \mathrm{~h}$ or $45 \mathrm{~h}$ for pyrene. Prolonged incubation was avoided to limit unwanted degradation of oxidation products. These products were recovered from the aqueous phase by solid-phase extraction and further purified as described below.

\section{Identification of the fluoranthene oxidation products}

The oxidation products formed upon incubation of fluoranthene with Bphn12 were separated by reverse-phase HPLC. Four UV-absorbing fractions displaying retention times ranging between 13 min (Fraction F1) and 28 min (F4) were collected. Fraction F1 essentially consisted of a single compound, yellow in color, which was further purified by thin layer chromatography. This product, designated as FP1, was by far the major product formed as judged from HPLC and TLC analysis of the crude extract. It showed an absorbance spectrum

21 with three maxima in the UV range and a broad maximum around $442 \mathrm{~nm}$ (see Fig S1A in 22 supplemental material). Fraction F2 was a mixture including some FP1 product (overlap), a monohydroxyfluoranthene with a GC retention time of 20,41 min (Table 1), and a compound

24 with a distinctive $R_{f}$ of 0.45 by TLC, which exhibited a mass spectrum typical of a 25 dihydrodiol (Table 1). This product was recovered in small amounts, thus precluding 
1 determination of the hydroxyl positions by NMR. Its UV absorbance spectrum, with maxima 2 at 232, 260 and $268 \mathrm{~nm}$ and a shoulder at $250 \mathrm{~nm}$, was clearly distinct from that of the cis 2,33 dihydrodiol, although the two dihydrodiols showed almost identical GC retention times 4 (Table 1 and see below). Based on these properties, and taking into account published data on 5 fluoranthene metabolism (Kweon et al. 2007; Lopez et al. 2006; Story et al. 2001), we 6 tentatively propose that this dihydrodiol carries hydroxyls on the $\mathrm{C} 1$ and $\mathrm{C} 2$ positions.

7 Fraction F3 mainly contained a compound with a TLC $R_{f}$ of 0.35 , which displayed UV 8 absorbance maxima at 230, 250, 260 and $290 \mathrm{~nm}$, and mass spectral features that identified it 9 as another dihydrodiol (Table 1). Its properties perfectly matched those of the previously characterized cis-2,3-dihydroxy-2,3-dihydrofluoranthene (Rehmann et al. 2001). The last HPLC fraction (F4) contained a single product, which showed the highest $R_{f}$ upon TLC (0.82). This compound exhibited UV absorbance (Fig. S1B) and GC/MS properties that closely matched those of 8-hydroxyfluoranthene (Table 1; (Jouanneau et al. 2006)).

The FP1 product turned out to be unstable and was converted to 8-hydroxyfluoranthene upon heating, as evidenced by UV absorbance recordings and TLC analysis. As a consequence of 16 its instability, analysis of FP1 by GC/MS almost exclusively yielded the 8hydroxyfluoranthene spectrum. Nevertheless, GC/MS analysis of a concentrated FP1 sample allowed the detection of a third diol with a $R_{t}$ of $20.30 \mathrm{~min}$ (Table 1).

${ }^{1} \mathrm{H}-\mathrm{NMR}$ analysis of the FP1 product revealed the presence of three mutually coupling groups identifying eight aromatic protons, two carbinol protons and two protons of hydroxyl groups.

21 The ${ }^{13} \mathrm{C}-\mathrm{NMR}$ and ${ }^{1} \mathrm{H},{ }^{13} \mathrm{C}-\mathrm{HMQC}$ spectra further indicated that ten carbons carried a proton, 22 two of which had a specific $\mathrm{sp}^{3}$ carbon chemical shift. The set of NMR assignments for this compound is summarized in Table 2. The chemical shifts at 4.55 and $4.95 \mathrm{ppm}$ were assigned to the $\mathrm{H}_{8}$ and $\mathrm{H}_{7}$ carbinol protons, respectively. Specifically, the value of the coupling constant between these vicinal protons $(6.5 \mathrm{~Hz})$ indicated that they were bound in a cis 
1 conformation (Jerina et al, 1984 ; Rehmann et al, 2001). We therefore conclude that the main

2 product generated by the CHY-1 dioxygenase from fluoranthene was the cis-7,8-dihydroxy-

3 7,8-dihydrofluoranthene.

4 In a previous study, the instability of this diol led us to erroneously conclude that the purified

5 CHY-1 dioxygenase converted fluoranthene to 8-hydroxyfluoranthene, based on GC/MS data

6 (Jouanneau et al. 2006). In fact, the absorbance of the product formed by the RHD complex in

7 vitro was quasi identical to that of the purified 7,8-dihydrodiol (Fig. S1A) and this was so,

8 because the other oxidation products identified above were produced in very low amounts at

9 least at the scale of our in vitro enzymatic reaction. Consistent with this observation, we

10 estimated that the recovery of purified cis 7,8-dihydrodiol from the Bphn12 culture (8.5

$11 \mu$ moles based on $A_{334}$ and $\varepsilon_{334}=10,100 \mathrm{M}^{-1} \mathrm{~cm}^{-1}$; see Methods) was 270-fold higher than that

12 of the cis 2,3-dihydrodiol (31.2 nmoles), the dihydrodiol that ranked second by abundance in

13 the culture extract. The conversion yields of fluoranthene into dihydrodiols are reported in

14 Table 1.

15 Hence, the biotransformation of fluoranthene by strain CHY-1 RHD essentially yielded one 16 predominant dihydrodiol hydroxylated on $\mathrm{C} 7$ and $\mathrm{C} 8$, and two minor dihydrodiols 17 hydroxylated on the $\mathrm{C} 2-\mathrm{C} 3$ and, presumably, the C1-C2 positions (Fig. 1A). The two 18 monohydroxy-fluoranthene also detected, one of which derived from the 7,8-dihydrodiol, 19 probably arose from spontaneous dehydration reactions.

21 Characterization of oxidation products generated from benz[a]anthracene and chrysene

22 The oxidation products found in Bph12 cultures upon incubation with BaA were extracted 23 and subjected to HPLC separation. Two main UV-absorbing fractions were obtained noted 24 BA1 and BA2, which were further analyzed by TLC. The BA1 fraction was developed with a 25 solvent consisting of $10 \%$ methanol in chloroform, resulting in the separation of a major 
1 compound with a $R_{f}$ of 0.165 . The UV absorbance spectrum of this compound is shown in

2 supplementary figure S2D. GC/MS analysis of the tms derivate of this compound gave a

3 molecular mass of 584 and a spectrum consistent with a bis-cis-dihydrodiol or tetraol (Table

4 1). Two additional components with higher $R_{t}(24.43$ and $25.01 \mathrm{~min})$ and lower relative 5 abundance showed a molecular mass of $494\left[584-\mathrm{OHSi}\left(\mathrm{CH}_{3}\right)_{3}\right]$ and a mass spectrum that 6 identified them as hydroxylated dihydrodiol derivatives of $\mathrm{BaA}$ (data not shown). These 7 compounds were probably dehydration products of the tetraol, which probably formed during derivatization step prior to GC/MS analysis.

9 The ${ }^{1} \mathrm{H}-\mathrm{NMR}$ spectrum of the BA1 product featured three mutually coupling groups indicating the presence of eight aromatic protons, four carbinol protons and four protons of 11 hydroxyl groups. 2D ${ }^{1} \mathrm{H},{ }^{1} \mathrm{H}-\mathrm{COSY}$, and ${ }^{1} \mathrm{H},{ }^{1} \mathrm{H}-\mathrm{NOESY}$ experiments allowed to define the 12 succession of these protons and therefore to assign all the chemical shifts. The ${ }^{13} \mathrm{C}$-NMR and the ${ }^{1} \mathrm{H},{ }^{13} \mathrm{C}$-HMQC spectra showed that twelve carbons carried a proton, four of which had a specific $\mathrm{sp}^{3}$ carbon chemical shift. The set of NMR assignments for this compound is summarized in Table 3. The chemical shifts at 4.36, 4.63, 4.77 and $5.28 \mathrm{ppm}$ were assigned to the $\mathrm{H}_{10}, \mathrm{H}_{2}$ non-benzylic, and the $\mathrm{H}_{11}, \mathrm{H}_{1}$ benzylic protons, respectively. The values of the two coupling constants between the carbinol protons, both at $5.4 \mathrm{~Hz}$, indicated that the vicinal protons had a cis conformation (Jerina et al, 1984).

In addition, no $\mathrm{W}$ coupling was observed between the allylic carbinol proton $\mathrm{H}_{2}$ and the vinylic proton $\mathrm{H}_{4}$, suggesting that the allylic hydroxyl group had a pseudo-axial conformation.

21 Likewise, no coupling was detected between the allylic carbinol proton $\mathrm{H}_{10}$ and the vinylic 22 proton $\mathrm{H}_{8}$ so that the allylic hydroxyl group would also be in a pseudo-axial conformation. 23 This analysis is confirmed by the coupling value of $4.5 \mathrm{~Hz}$ between the allylic carbinol proton $24 \mathrm{H}_{10}$ and the vinylic proton $\mathrm{H}_{9}$. Indeed, it has been shown that the magnitude of the coupling corresponded to the case where the allylic hydroxyl group had a pseudo-axial conformation 
(Jerina et al. 1976).

2 Altogether, our NMR and GC/MS data showed that the BA1 compound was a BaA tetraol

3 with two pairs of hydroxyl groups both in cis conformation, occupying the carbon positions

4 C1-C2 and C10-C11. Moreover, NMR data provided evidence that the hydroxyl groups on C2

5 and C10 would preferentially adopt a pseudo-axial conformation as depicted in Fig. 2.

6 The second HPLC fraction (BA2) was found to contain three dihydrodiols by GC/MS (Table

7 1). The most abundant one (60-72\%) could be isolated as a pure component upon further reverse-phase HPLC, taking advantage of its slightly shorter retention time compared to that of the other diols. It was identified as cis-1,2-BaA dihydrodiol based on its UV absorbance spectrum (Fig. S2A) and mass spectral data that matched those previously reported for this compound (Jerina et al., 1984). The other two diols were successfully separated by normal 12 phase HPLC separation (Rt of 24.5 and $28 \mathrm{~min}$, respectively). They showed distinctive absorbance features in the UV range (Fig. S2B and S2C) and were identified based on the same criteria as above as the cis-8,9- and cis-10,11-dihydrodiols of BaA. The four BaA hydroxylated products generated by the CHY-1 RHD are represented in Fig. 1B.

Chrysene biotransformation by Bphn12 gave essentially two products that could be easily separated by HPLC. The product with the shortest retention time yielded a single band by TLC ( $R_{f}: 0.145$ with chloroform/methanol, 9/1 as solvent), and gave a UV absorbance spectrum with maxima at 264, 274, 338, 350, $368 \mathrm{~nm}$ (Fig. S3B). As deduced from the mass spectrum of the tms derivative and its molecular mass $\left(\mathrm{M}^{+}=584\right)$, this product was identified as a bis-cis-dihydrodiol (Table 1). As observed for the BaA tetraol, two additional GC/MS peaks with a molecular mass of 494 were also detected (data not shown). Likewise, these compounds probably formed by dehydration of the unstable tetraol.

24 Further characterization of this tetraol by NMR showed that it resulted from two cis25 dihydroxylation of chrysene (Table 4). In all NMR spectra (1D and $2 \mathrm{D}{ }^{1} \mathrm{H}-$ or $\left.{ }^{13} \mathrm{C}-\mathrm{NMR}\right)$, the 
1 number of signals was half that of the protons or carbon atoms (except for quaternary

2 carbons), indicating that the molecule had a symmetrical structure (Table 4). The chemical

3 shifts at 4.53 and 5.16 ppm were assigned to the $\mathrm{H}_{3}\left(\right.$ or $\left.\mathrm{H}_{9}\right)$ and $\mathrm{H}_{4}\left(\right.$ or $\left.\mathrm{H}_{10}\right)$ carbinol protons,

4 respectively. A single coupling constant at $4.8 \mathrm{~Hz}$ was observed between the two pairs of

5 vicinal carbinol protons, indicating that both had a cis conformation.

6 Unlike what was observed for the BaA tetraol, the $\mathrm{W}$ coupling constant between the allylic

7 carbinol proton $\mathrm{H}_{3}\left(\right.$ or $\left.\mathrm{H}_{9}\right)$ and the vinylic proton $\mathrm{H}_{1}\left(\right.$ or $\left.\mathrm{H}_{7}\right)$ had a value of $3.9 \mathrm{~Hz}$, consistent

8 with a pseudo-equatorial conformation of the hydroxyl group. Accordingly, a coupling

9 constant $<2 \mathrm{~Hz}$ was found between the allylic carbinol proton $\mathrm{H}_{3}$ and the vinylic proton $\mathrm{H}_{2}$,

10 thus supporting the suggested conformation for the hydroxyls on C3 and C9.

11 In conclusion, our data provide evidence that one of the major product formed upon chrysene

12 oxygenation by strain CHY-1 RHD was the cis,cis-3,4,9,10-tetrahydroxy-3,4,9,10-

13 tetrahydrochrysene. Furthermore, NMR data indicate that the hydroxyl groups bound to C3

14 and C9 would be in a pseudo equatorial configuration.

The second HPLC fraction was found to consist of a single compound showing a UV absorbance spectrum (Fig. S3A) and mass spectral data $\left(\mathrm{M}^{+}=406\right.$, tms derivative), which closely matched those of the previously described cis-3,4-dihydroxy 3,4-dihydrochrysene (Boyd et al. 1997). From the UV absorbance of the purified fraction $\left(\varepsilon_{27 s}=58,650 \mathrm{M}^{-1} \cdot \mathrm{cm}^{-1}\right)$, approx. 168 nmoles of cis-3,4-dihydrodiol was recovered per liter of culture. To explain the 21 occurrence of the teraol, it seems likely that the dioxygenase first attacked the substrate on the C3-C4 positions, then used the dihydrodiol as substrate to form the tetraol (Fig. 1C).

24 Biotransformation of pyrene and benzo[a]pyrene

25 Following incubation of a Bphn12 culture with pyrene, a UV detectable product (260 nm) 
1 appeared in the extract of the aqueous phase. This product was purified by HPLC and analyzed by its UV absorbance spectroscopy. Its spectrum exhibited maxima at 220, 262 and $298 \mathrm{~nm}$ and closely resembled that of the cis 4,5-pyrene dihydrodiol, which we had previously identified as generated by another RHD from a pyrene-degrading Mycobacterium strain (Krivobok et al. 2003). GC/MS analysis confirmed the identification of the product as its $R_{t}$ and mass spectrum matched those of the cis 4,5-pyrene dihydrodiol. Compared to the other substrates studied herein, pyrene yielded a relatively small amount of diol (only about $15 \mathrm{nmoles} / \mathrm{L}$ culture based on GC/MS estimation) within an incubation time twice as long, suggesting that it was a poor substrate for strain CHY-1 RHD.

Benzo[a]pyrene $(\mathrm{BaP})$ was converted by Bphn12 cultures into one major product that gave a 11 single peak upon HPLC analysis. The UV absorbance spectrum of this compound (Fig. S4) was quite similar to that of cis-9,10-dihydroxy-9,10-dihydroBaP (Gibson et al., 1975). GC/MS analysis of the tms derivate was consistent with the dihydrodiol structure of the product (Table 1). Further analysis of the product after N-butylboronate derivatization yielded a major compound with the expected molecular mass of $352\left(R_{t}: 26.98 \mathrm{~min}\right)$ and a minor component with the same molecular mass and a higher $R_{t}(27.85 \mathrm{~min})$, which accounted for about $7 \%$ of the total amount of diols based on GC/MS peak area. Although the latter compound was too scarce to allow rigorous identification, it might be the cis-7,8-dihydrodiol previously found to be a secondary product formed from BaP by a similar RHD (Gibson et al., 1975). Based on the $A_{280}$ absorption of the $9,10-\mathrm{BaP}$ dihydrodiol, we estimated that the 21 Bphn12 culture produced approximately $72 \mathrm{nmoles} / \mathrm{L}$ of this diol.

\section{DISCUSSION}


1 naphthalene dioxygenase and showed that it could dihydroxylate two additional 4-ring PAHs,

2 pyrene and fluoranthene. Taking into account previous reports on this enzyme (Demaneche et

3 al. 2004; Jouanneau et al. 2006), its substrate range was thus extended to include nine PAHs

4 of the priority list made of up to 5 rings. Such a broad specificity is exceptional and most

5 likely due to the size and topology of its substrate-binding pocket, which is larger than that of

6 most known RHDs (Jakoncic et al. 2007a; Jakoncic et al. 2007b). The CHY-1 dioxygenase

7 shows close similarities with the biphenyl dioxygenase from Sphingobium yanoikuiyae B1,

8 the two enzymes sharing a high level of sequence identity (91.0\% and $96.6 \%$ for the alpha

9 and beta subunits, respectively), an almost identical 3D structure (Ferraro et al. 2007;

10 Jakoncic et al. 2007b) and a comparable propensity to attack HMW-PAHs ((Jouanneau et al.

11 2006; Yu et al. 2007) and references cited below). Yet, there are differences of substrate

12 preference, biphenyl being the best substrate of the latter enzyme, whereas naphthalene was preferentially utilized by strain CHY-1 RHD. This suggested that subtle structural differences accounted for the observed changes in substrate preference.

With the purified CHY-1 dioxygenase, it was observed that the rate of substrate oxidation was

16 inversely correlated with the substrate size (Jouanneau et al. 2006). In the present study, we

17 found that substrates of similar size underwent quite different oxidation rates. From the conversion yields of the studied PAHs (Table 1), the substrate preference of the CHY-1 dioxygenase would respect the following order: fluoranthene $>$ benz[a]anthracene $>$ chrysene

$20>$ benzo[a]pyrene $>$ pyrene. This observation indicated that steric constraints other than those

21 merely due to substrate size affected the rate of the dioxygenation reaction. In PAH-degrading 22 Mycobacteria, multiple RHDs with relatively narrow specificities were found. 23 Mycobacterium sp. strain 6PY1 synthesized two RHDs showing marked preferences for 24 phenanthrene and pyrene, respectively (Krivobok et al. 2003). In M. vanbalenii PYR-1, two RHDs that exhibited narrow specificities for pyrene and fluoranthene, respectively, were 
1 subjected to molecular modeling as a means to try to correlate substrate specificity with the

2 shape of the substrate-binding pocket (Kweon et al. 2010). However, 3D structure

3 determination is needed to draw relevant conclusions on structure-function correlations

4 regarding these enzymes.

5 Our results indicate that fluoranthene is a good substrate for the CHY-1 dioxygenase relative

6 to other 4-ring PAHs in accordance with previous data obtained with the purified enzyme

7 (Jouanneau et al. 2006). The enzyme proved capable of attacking the four-ring hydrocarbon

8 on three positions, indicating that fluoranthene can enter the substrate-binding pocket in three

9 different ways. Likewise, $\mathrm{BaA}$ gave rise to three dihydrodiols, which were found to

10 correspond to three possible orientations of the substrate at the active site as predicted from

11 docking experiments with the 3D structure of the oxygenase component (Jakoncic et al.,

12 2007a). With fluoranthene as substrate, however, the orientation yielding the cis-7,8-

13 dihydrodiol was highly preferred since other diols were found in proportions $<1 \%$. Despite

14 the fact that strain CHY-1 cannot grow on fluoranthene (Willison , 2004), our findings

15 suggest that it might be degraded further by cleavage of the aromatic ring adjacent to the C7-

16 C8 bond. In contrast, Mycobacterium species preferentially metabolize fluoranthene by

17 another route involving an initial attack on the C2-C3 carbon positions (Rehmann et al., 2001;

18 Lopez et al., 2006; Kweon e al., 2007). This difference is certainly related to the catalytic

19 properties of the RHD oxidizing fluoranthene in PAH-degrading Mycobacteria, which, in $M$.

20 vanbalenii PYR-1, has been shown to produce almost exclusively the cis 2,3-dihydrodiol

21 (Kim et al., 2006).

22 BaA also appeared as a fairly good substrate for strain $\mathrm{CHY}-1$ dioxygenase as judged from 23 the recovery of oxidation products. The enzymatic reaction yields three dihydrodiols and a

24 tetraol, which has been characterized as cis,cis-1,2,10,11-tetrahydroxy 1,2,10,1125 tetrahydroBaA based on MS and NMR data. The absolute configuration of this compound 
1 could not be determined, but it seems likely that the pairs of hydroxyls bonded on C1-C2 and

2 C10-C11 be oriented on opposite faces of the molecule (Fig. 2). This assumption is deduced

3 from our previous observation that the tetraol is produced by the purified RHD either from the

4 1,2- or the 10,11-dihydrodiol, which have unique configurations (Fig. 1B; Jouanneau et al., 5 2006). Among other RHDs able to oxidize BaA, the $S$. yanoikuyae B1 RHD mostly resembles

6 the CHY-1 enzyme in that it produces the same three dihydrodiols from BaA (Jerina et al., 7 1984). Formation of the relevant BaA tetraol was however not reported, although strain B1 8 RHD was shown to form another tetraol from the BaA 5,6-dihydrodiol (Boyd et al., 2006).

9 Besides, the RHD from both strains were shown to convert another 4-ring diol, chrysene 3,4dihydrodiol, to a tetraol hydroxylated in positions 3,4,9 and 10 (Fig. 2C; Boyd et al., 1999).

11 Hence, there are strong apparent similarities in catalytic activity and regioselectivity between 12 the two enzymes, which likely reflect their closely related structures. As previously underlined for the strain B1 RHD, the hydroxylation of 4-ring substrates preferentially occurs on the bay sides of the molecules (Boyd et al. 1999), a propensity certainly linked to the form and topology of the substrate-binding pocket, which determines the orientation of the 16 substrate and thereby, the enzyme regioselectivity (Jakoncic et al., 2007a). The initial attack 17 of BaA in Mycobacterium strains appears to involve RHDs with a different selectivity. Strain RGII-135 was found to convert BaA into two dihydrodiols, the most abundant of which being hydroxylated on C5-C6 (Schneider et al., 1996). In M.. vanbalenii PYR-1, the two RHDs able to attack HMW-PAHs exhibited little activity towards BaA, and converted it to the 5,6- and 21 10,11-dihydrodiols (Kim 2006, Kweon 2010).

22 In this work, we observed that the CHY-1 dioxygenase showed a better activity with BaP as 23 substrate than with pyrene, although the latter substrate is smaller. Considering the structural 24 similarities between the two polyaromatic substrates, this result is intriguing and might be 25 explained by assuming that the additional ring in BaP helps this substrate to be processed at th 
1 enzyme active site. Accordingly, docking experiments performed with the dioxygenase 3D

2 structure previously showed that $\mathrm{BaP}$ fitted into the substrate-binding pocket in only one

3 position, where the additional ring was proximal to the iron atom at the active site (Jakoncic

4 et al. 2007a). This position of $\mathrm{BaP}$ explains why it is preferentially hydroxylated on the C9-

$5 \mathrm{C} 10$ positions. On the other hand, it is conceivable that the access of the compact 4-ring

6 molecule of pyrene to the enzyme active site might be hindered by steric constraints due to

7 the narrow shape of the substrate-binding pocket near the iron atom. Beyond this trait

8 particular to the CHY-1 dioxygenase, examples of RHDs more active on HMW-PAHs than

9 on 2- or 3-ring hydrocarbons have been described, especially in Mycobacterium 6PY1

10 (Krivobok et al. 2003) and M. vanbalenii PYR-1 (Kweon et al. 2010)..

11 The broad specificity of the dioxygenase described in this study, especially its ability to attack

12 HMW-PAHs, makes it a valuable biocatalyst, potentially useful in bioremediation of 13 contaminated sites. The enzyme is also of interest in that it converts aromatic substrates to 14 pure cis-dihydrodiols (or bis,cis-dihydrodiols). In this respect, it shares with the S. yanoikuyae 15 B1 dioxygenase, the ability to convert 3- and 4-ring compounds to chiral dihydrodiols or 16 tetraols, some of which might be useful for the synthesis of fine chemicals and 17 pharmaceuticals (Boyd et al. 2006; Boyd et al. 2008; Boyd et al. 1999).

\section{Acknowledgements}

20 The authors are grateful to the CNRS and Univ Grenoble Alpes for funding. This work was 21 also supported in part by a grant from the ANR "programme Labex" (ARCANE project $\mathrm{n}^{\circ}$ 22 ANR-11-LABX-003).

24 Conflict of interest The authors declare that they have no competing interests. 


\section{References}

2 Bamforth SM, Singleton I (2005) Bioremediation of polycyclic aromatic hydrocarbons: current knowledge and future directions. J Chem Technol Biotechnol 80:723-736

Boyd DR, Sharma ND, Agarwal R, Resnick SM, Schocken MJ, Gibson DT, Sayer JM, Yagi H, Jerina DM (1997) Bacterial dioxygenase-catalysed dihydroxylation and chemical resolution routes to enantiopure cis-dihydrodiols of chrysene. J Chem Soc-Perkin Trans 1(11):1715-1723

Boyd DR, Sharma ND, Belhocine T, Malone JF, McGregor S, Allen CCR (2006) Dioxygenase-catalysed dihydroxylation of arene cis-dihydrodiols and acetonide derivatives: a new approach to the synthesis of enantiopure tetraoxygenated bioproducts from arenes. Chem Commun(47):4934-4936

Boyd DR, Sharma ND, Coen GP, Hempenstall F, Ljubez V, Malone JF, Allen CCR, Hamilton JTG (2008) Regioselectivity and stereoselectivity of dioxygenase catalysed cis-dihydroxylation of mono- and tri-cyclic azaarene substrates. Org Biomol Chem $6(21): 3957-3966$

Boyd DR, Sharma ND, Hempenstall F, Kennedy MA, Malone JF, Allen CCR, Resnick SM, Gibson DT (1999) bis-cis-Dyhydrodiols: a new class of metabolites resulting from biphenyl dioxygenase-catalyzed sequential asymetric cis-dihydroxylation of polycyclic arenes and heteroarenes. J Org Chem 64: 4005-4011

Demaneche S, Meyer C, Micoud J, Louwagie M, Willison JC, Jouanneau Y (2004) Identification and functional analysis of two aromatic ring-hydroxylating dioxygenases from a Sphingomonas strain degrading various polycyclic aromatic hydrocarbons. Appl Environ Microbiol 70:6714-6725

Doyle E, Muckian L, Hickey AM, Clipson N (2008) Microbial PAH Degradation. Adv Appl Microbiol 65:27-66 
1 Ferraro DJ, Brown EN, Yu CL, Parales RE, Gibson DT, Ramaswamy S (2007) Structural investigations of the ferredoxin and terminal oxygenase components of the biphenyl 2,3-dioxygenase from Sphingobium yanoikuyae BI. BMC Struct Biol 7(10)

Gibson DT, Mahadevan V, Jerina DM, Yogi H, Yeh HJ (1975) Oxidation of the carcinogens benzo [a] pyrene and benzo [a] anthracene to dihydrodiols by a bacterium. Science 189(4199):295-297

Heitkamp MA, Freeman JP, Miller DW, Cerniglia CE (1988) Pyrene degradation by a Mycobacterium sp.: identification of ring oxidation and ring fission products. Appl Environ Microbiol 54:2556-2565

Jakoncic J, Jouanneau Y, Meyer C, Stojanoff V (2007a) The catalytic pocket of the ringhydroxylating dioxygenase from Sphingomonas CHY-1. Biochem Biophys Res Commun 352:861-866

Jakoncic J, Jouanneau Y, Meyer C, Stojanoff V (2007b) The crystal structure of the ringhydroxylating dioxygenase from Sphingomonas CHY-1. Febs J 274:2470-2481

Jerina DM, Selander H, Yagi H, Wells MC, Davey JF, Mahadevan V, Gibson DT (1976) Dihydrodiols from anthracene and phenathrene. J Am Chem Soc 98(19):5988-5996

Jerina DM, Vanbladeren PJ, Yagi H, Gibson DT, Mahadevan V, Neese AS, Koreeda M, Sharma ND, Boyd DR (1984) Synthesis and absolute-configuration of the bacterial cis-1,2-dihydrodiol, cis-8,9-dihydrodiol, and cis-10,11-dihydrodiol metabolites of benz[a]anthracene formed by a strain of Beijerinckia. J Org Chem 49:3621-3628

Jouanneau Y, Martin F, Krivobok S, Willison JC (2011) Ring-hydroxylating dioxygenases involved in PAH biodegradation : structure, function and biodiversity. In: Koukkou AI (ed) Microbial bioremediation of non metals: current research. Caister Academic Press, Norflok, UK, pp 149-175 
1 Jouanneau Y, Meyer C, Jakoncic J, Stojanoff V, Gaillard J (2006) Characterization of a naphthalene dioxygenase endowed with an exceptionally broad substrate specificity toward polycyclic aromatic hydrocarbons. Biochemistry 45(40):12380-12391

Juhasz AL, Naidu R (2000) Bioremediation of high molecular weight polycyclic aromatic hydrocarbons: a review of the microbial degradation of benzo a pyrene. Intern Biodeter Biodeg 45:57-88

Karlsson A, Parales JV, Parales RE, Gibson DT, Eklund H, Ramaswamy S (2003) Crystal structure of naphthalene dioxygenase: side-on binding of dioxygen to iron. Science 299(5609):1039-1042

Kim SJ, Kweon O, Jones RC, Freeman JP, Edmondson RD, Cerniglia CE (2007) Complete and integrated pyrene degradation pathway in Mycobacterium vanbaalenii PYR-1 based on systems biology. J Bacteriol 189:464-472

Krivobok S, Kuony S, Meyer C, Louwagie M, Willison JC, Jouanneau Y (2003) Identification of pyrene-induced proteins in Mycobacterium sp. 6PY1 : Evidence for two ring-hydroxylating dioxygenases. J Bacteriol 185:3828-3841

Kunihiro M, Ozeki Y, Nogi Y, Hamamura N, Kanaly RA (2013) Benz[a]anthracene biotransformation and production of ring fission products by Sphingobium sp. strain KK22. Appl Environ Microbiol 79:4410-4420

Kweon O, Kim S-J, Baek S, Chae J-C, Adjei M, Baek D-H, Kim Y-C, Cerniglia C (2008) A new classification system for bacterial Rieske non-heme iron aromatic ringhydroxylating oxygenases. BMC Biochem 9:11

Kweon O, Kim S-J, Jones RC, Freeman JP, Adjei MD, Edmondson RD, Cerniglia CE (2007) A polyomic approach to elucidate the fluoranthene-degradative pathway in Mycobacterium vanbaalenii PYR-1. J Bacteriol 189:4635-4647 
1 Kweon O, Kim SJ, Freeman JP, Song J, Baek S, Cerniglia CE (2010) Substrate specificity and structural characteristics of the novel Rieske nonheme iron aromatic ringhydroxylating oxygenases NidAB and NidA3B3 from Mycobacterium vanbaalenii PYR-1. mBio 1(2) e00135-10-e00135-20

Lopez Z, Vila J, Minguillon C, Grifoll M (2006) Metabolism of fluoranthene by Mycobacterium sp strain AP1. Appl Microbiol Biotechnol 70:747-756

Lu X-Y, Zhang T, Fang HH-P (2011) Bacteria-mediated PAH degradation in soil and sediment. Appl Microbiol Biotechnol 89:1357-1371

Lyu Y, Zheng W, Zheng T, Tian Y (2014) Biodegradation of polycyclic aromatic hydrocarbons by Novosphingobium pentaromativorans US6-1. Plos One 9(7) doi:10.1371/journal.pone.0101438

Maeda AH, Nishi S, Hatada Y, Ozeki Y, Kanaly RA (2014) Biotransformation of the highmolecular weight polycyclic aromatic hydrocarbon (PAH) benzo k fluoranthene by Sphingobium sp strain KK22 and identification of new products of non-alternant PAH biodegradation by liquid chromatography electrospray ionization tandem mass spectrometry. Microbial Biotechnol 7:114-129

Nam JW, Nojiri H, Yoshida T, Habe H, Yamane H, Omori T (2001) New classification system for oxygenase components involved in ring- hydroxylating oxygenations. Biosci Biotechnol Biochem 65:254-263.

Parales RE, Resnick SM (2006) Aromatic ring hydroxylating dioxygenases. In: Ramos JL, Levesque RC (eds) Pseudomonas. vol 4. Springer, pp 287-340

Peng RH, Xiong AS, Xue Y, Fu XY, Gao F, Zhao W, Tian YS, Yao QH (2008) Microbial biodegradation of polyaromatic hydrocarbons. FEMS Microbiol Rev 32(6):927-55 
1 Rehmann K, Hertkorn N, Kettrup AA (2001) Fluoranthene metabolism in Mycobacterium sp strain KR20: identity of pathway intermediates during degradation and growth. Microbiology-Sgm 147:2783-2794

Schneider J, Grosser R, Jayasimhulu K, Xue W, Warshawsky D (1996) Degradation of pyrene, benz[a]anthracene, and benzo[a]pyrene by Mycobacterium sp. strain RJGII135, isolated from a former coal gasification site. Appl Environ Microbiol 62:13-19

Story SP, Parker SH, Hayasaka SS, Riley MB, Kline EL (2001) Convergent and divergent points in catabolic pathways involved in utilization of fluoranthene, naphthalene, anthracene, and phenanthrene by Sphingomonas paucimobilis var. EPA505. J Ind Microbiol Biotechnol 26(6):369-382

Vila J, Lopez Z, Sabate J, Minguillon C, Solanas AM, Grifoll M (2001) Identification of a novel metabolite in the degradation of pyrene by Mycobacterium sp strain AP1: Actions of the isolate on two- and three-ring polycyclic aromatic hydrocarbons. Appl Environ Microbiol 67:5497-5505

Willison JC (2004) Isolation and characterization of a novel sphingomonad capable of growth with chrysene as sole carbon and energy source. FEMS Microbiol Lett 241:143-150

Yu CL, Liu W, Ferraro DJ, Brown EN, Parales JV, Ramaswamy S, Zylstra GJ, Gibson DT, Parales RE (2007) Purification, characterization, and crystallization of the components of a biphenyl dioxygenase system from Sphingobium yanoikuyae B1. J Ind Microbiol Biotechnol 34(4):311-324 
A<smiles>O[C@H]1C=Cc2cc3ccc4c(c3cc2[C@@H]1O)[C@@H](O)[C@H](O)C=C4</smiles>

C

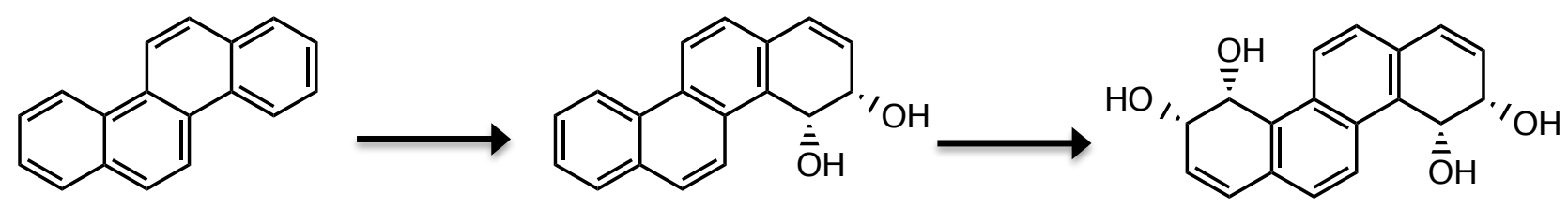

D<smiles></smiles><smiles>O[C@H]1c2cccc3cccc(c23)[C@H]1O</smiles>

E<smiles>O[C@H]1C=Cc2cc3ccc4cccc5ccc(c2-c3c54)[C@@H]1O</smiles>

Fig. 1, Jouanneau et al.<smiles>c1ccc2c(c1)cc1ccc3cccc4ccc2c1c34</smiles>

Fig 2 Jouanneau et a<smiles>O[C@H]1C=Cc2c(cc3ccc4cccc5ccc2c3c45)[C@H]1O</smiles> 


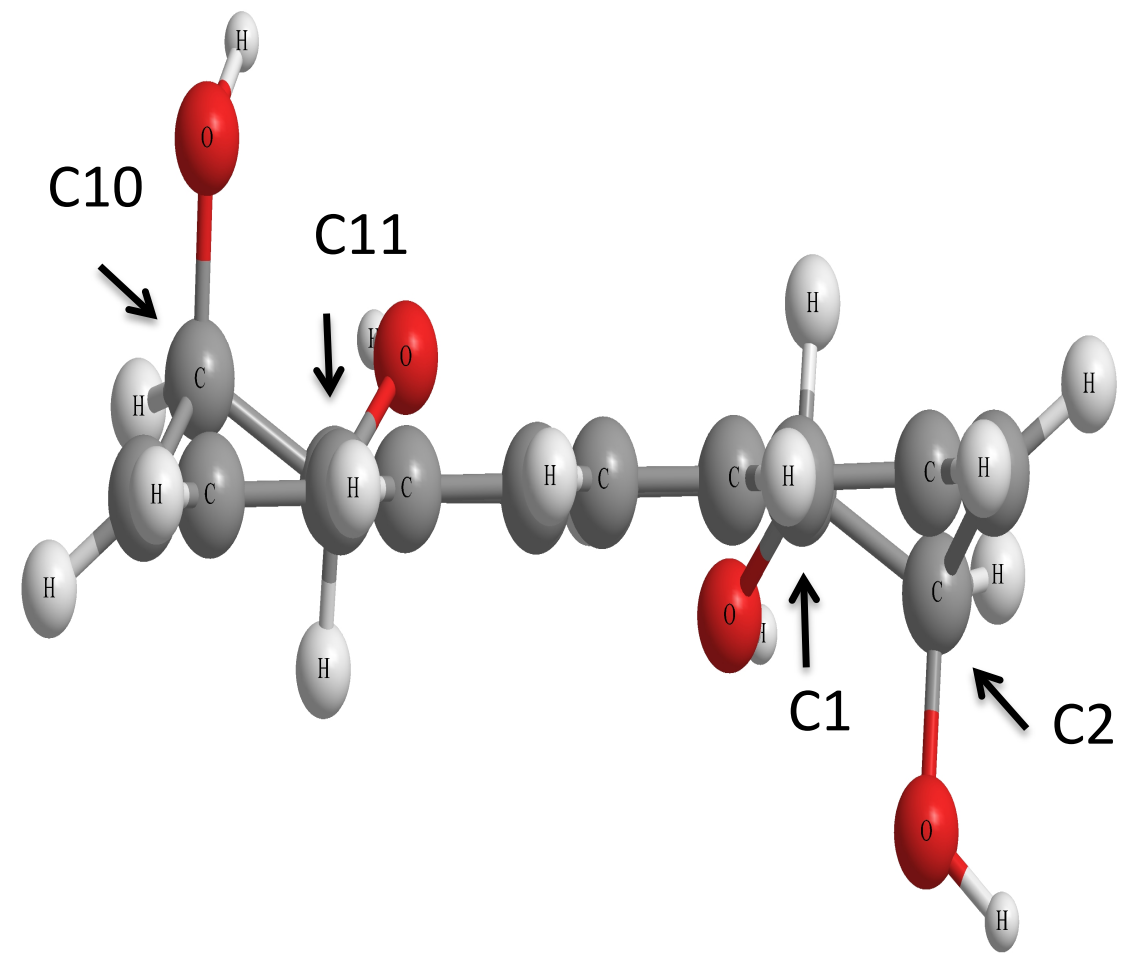

Fig. 2, Jouanneau et al. 
Supplemental figures to :

Applied Microbiology and Biotechnology

Dihydroxylation of four- and five-ring aromatic hydrocarbons by the naphthalene dioxygenase from Sphingomonas CHY-1

Yves Jouanneau $^{1,2,3}$, Christine Meyer ${ }^{1,2,3}$, and Nicolas Duraffourg ${ }^{1,2,3}$

${ }^{1}$ CEA, DSV, iRTSV, Chimie et Biologie des Métaux, F-38054 Grenoble, France.

${ }^{2}$ CNRS, UMR 5249, Grenoble, France.

${ }^{3}$ Univ. Grenoble Alpes, F-38000 Grenoble, France

Corresponding author:

Yves Jouanneau

LCBM/iRTSV, CEA-Grenoble

F-38054 Grenoble Cedex 9, France.

Tel. : 33 (0)4 387843 10; Fax : 33 (0)4 38785487

Email : yves.jouanneau@cea.fr 


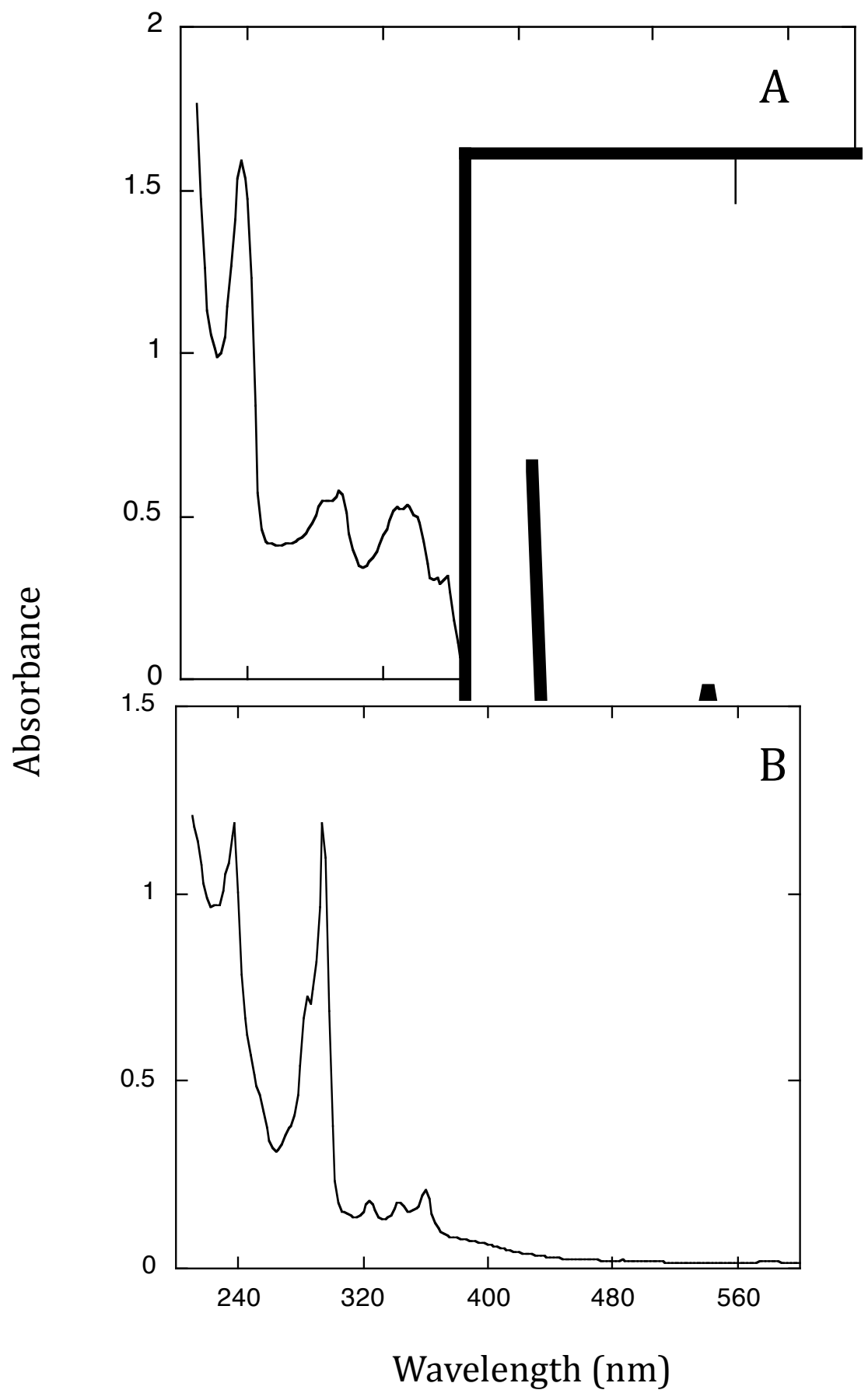

Figure S1 : Absorbance spectra of two main oxidation products of fluoranthene A; cis-7,8-dihydroxy-7,8-dihydrofluoranthene, $53 \mu \mathrm{M} ; \mathrm{B}$ : 8-hydroxyfluoranthene. Both spectra were recorded from solutions in acetonitrile. 

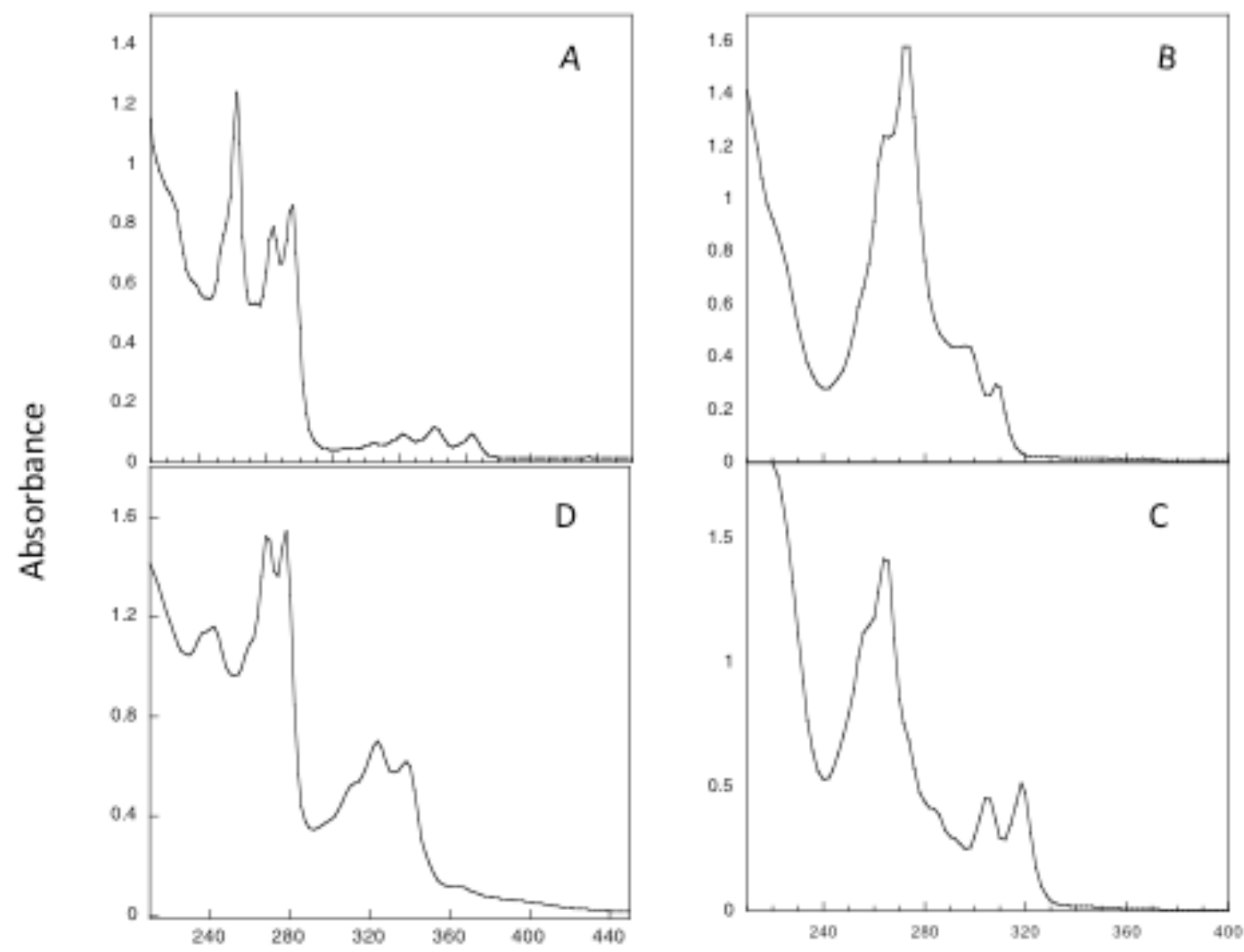

Wavelength $(\mathrm{nm})$

Figure S2: Absorbance spectra of benz[a]anthracene oxidation products

A: cis-1,2-dihydrodiol; B: cis-10,11-dihydrodiol; C: cis-8,9-dihydrodiol; D: cis,cis-1,2,10,11tetraol. All samples were in acetonitrile, with the dihydrodiol concentration being approx.. 40 $\mu \mathrm{M}$. 


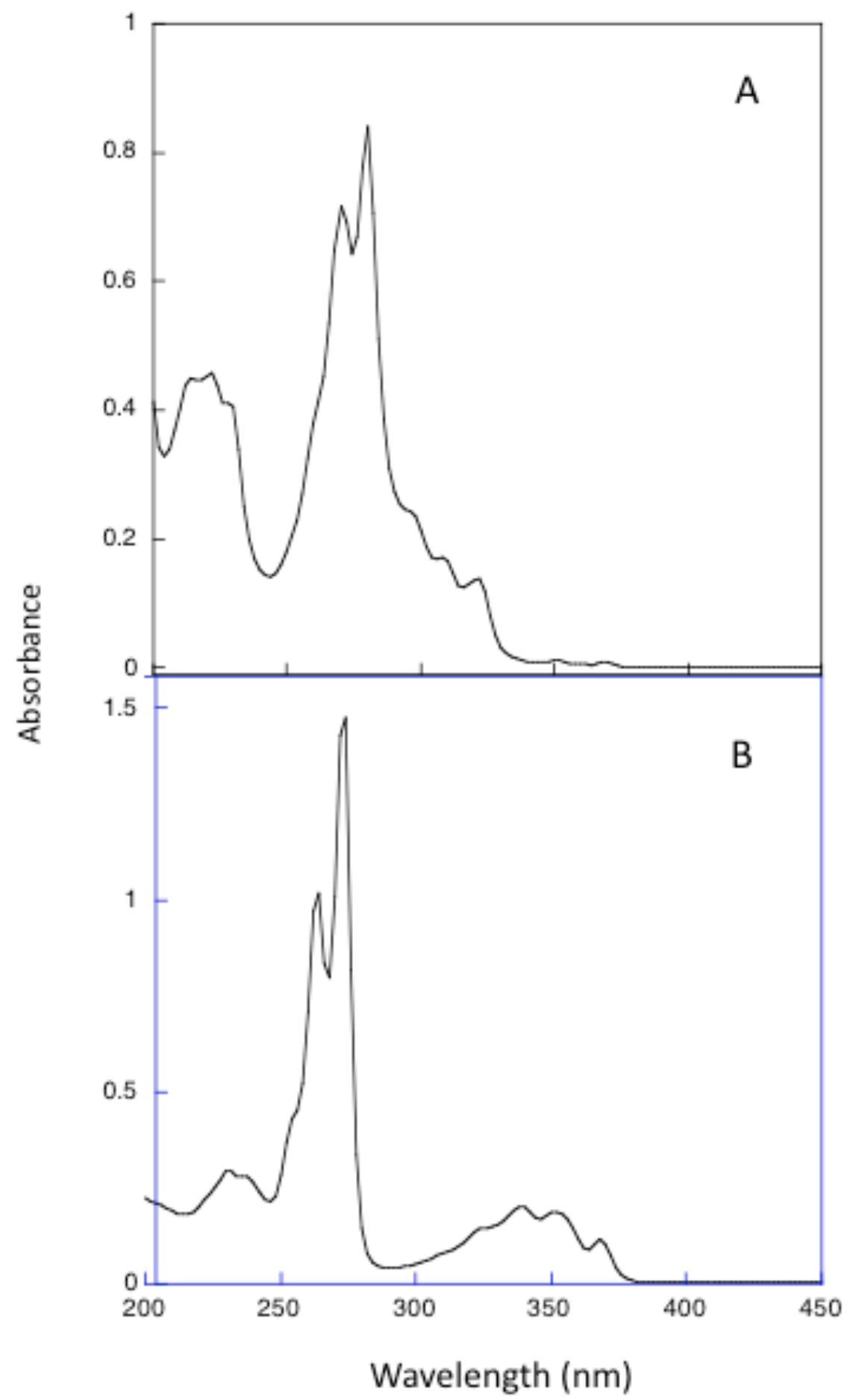

Figure S3: UV absorbance spectra of the dihydrodiol and tetraol generated from chrysene A: cis-3,4-dihydroxy-3,4-dihydrochrysene; B: cis,cis-3,4,9,10-chrysene tetraol. Samples were in acetonitrile. 


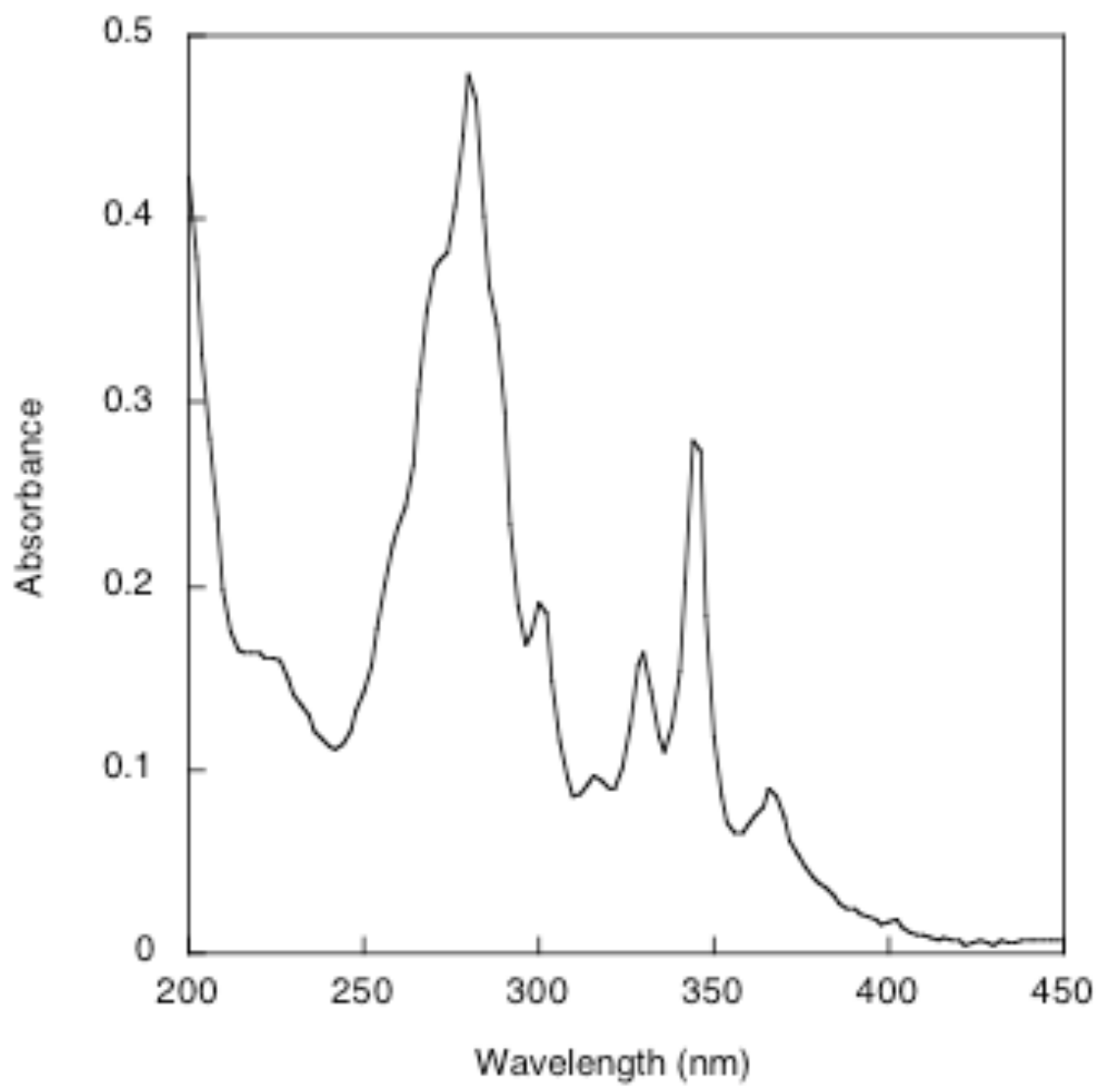

Figure S4: Absorbance spectrum of the cis-9,10-dihydrodiol formed from benzo[a]pyrene.

The sample concentration was $7.1 \mu \mathrm{M}$ in acetonitrile. 